\title{
Circadian Misalignment Leads to Changes in Cortisol Rhythms, Blood Biochemical Variables and Serum miRNA Profiles
}

\section{Guo-Bin Huang}

Sun Yat-Sen University

Huan Ma

Sun Yat-Sen University

Xi-Hui Gan

Sun Yat-Sen University

Si-Lin Li

Sun Yat-Sen University

Xiao-Hong Ma

Sun Yat-Sen University

Si-Yu Chen

Sun Yat-Sen University

Hui-Ying Yang

Sun Yat-Sen University

\section{Tuo-Yang Zhou}

China Institute of Marine Technology \& Economy

\section{Liang Zhang}

China Institute of Marine Technology \& Economy

Zhi-Qiang Tian

Bangladesh Institute of Marine Technology

\section{Xin Wang}

China Institute of Marine Technology \& Economy

Zhen Liao

China Institute of Marine Technology \& Economy

\section{Xin-Hai Zhu}

Sun Yat-Sen University

Hong-Mei Jiang

Sun Yat-Sen University

\section{Yong-Cong Shao}

Beijing Sport University

\section{Ye-Bing Yang}


General Hospital of People's Liberation Army: Chinese PLA General Hospital

Jin-Hu Guo ( $\square$ guojinhu@mail.sysu.edu.cn )

Sun Yat-Sen University https://orcid.org/0000-0003-4405-3959

\section{Research}

Keywords: circadian rhythm, shift work, entrainment, cortisol, blood biochemical parameter, miRNA

Posted Date: February 3rd, 2021

DOI: https://doi.org/10.21203/rs.3.rs-184041/v1

License: (1) This work is licensed under a Creative Commons Attribution 4.0 International License. Read Full License 


\section{Abstract}

Background: Circadian clock plays a critical role in synchronizing the inner molecular, metabolic and physiological processes to the environmental cues which cycle with a period of $24 \mathrm{~h}$. Misalignment in the circadian rhythms leads to decrease in adaptation, performance and increased risk of associated disorders. The non-24-h schedules and shift schedules are commonly used in maritime operations, both of which could result in disturbance in circadian rhythms. The comprehensive effects of these schedules remain to be further elucidated.

Methods: In this study, we firstly conducted an experiment in which the volunteers followed a 3-d rotary schedule with consecutive shift in sleep time (rotatory schedule), and analyzed the changes in salivary cortisol rhythms and blood variables. Next we conducted another experiment in which the volunteers followed an $8 \mathrm{~h}$ on and $4 \mathrm{~h}$ off schedule (non-24-h schedule) to compare the changes in blood/serum variables. Furthermore, the plasma miRNAs from the volunteers following the rotatory schedule were subject for miRNA sequencing.

Results: The rotatory schedule led to elevated levels of serum cortisol during the early stage, and the phase became delayed during the early and stages. The results show both of the schedules caused comprehensive changes in blood/serum biochemical variable, and consistently, the increase in phosphate levels. In addition, a subset of serum miRNAs targeting genes involved in circadian rhythms, sleep homeostasis and multiple important physiological processes or pathways were identified in the experiment with rotatory schedule.

Conclusions: This study revealed an increased stress during the rotatory schedule. Circadian misalignment caused by either non-24-h or rotatory schedule lead to extensive changes in blood/serum variables. As to the rotatory schedule, altered expression of serum miRNAs may account for the consequences of circadian misalignment. These findings would help understand the deleterious effects of shift schedules and optimize to enhance performance and welfare of the personnel working with similar schedules.

\section{Background}

Circadian clocks are ubiquitous in most of the organisms on earth, which play critical roles in synchronizing the internal physiology with the external cycling environment cues with a period of $24 \mathrm{~h} \mathrm{[1].}$ Environmental, genetic, physiological and pathological factors may cause disturbance in circadian rhythms [2]. In humans, circadian clock controls a broad variety of physiological, cognitive, emotional and behavioral features, and misalignment in circadian rhythms leads to detrimental effects on health and performance [3].

The oscillation of many human physiological variables is governed by circadian clock $[4,5]$. Not surprisingly, most blood biochemical contents are also under the circadian control, e.g., polymorphonuclears, lymphocytes, $\mathrm{Ca}^{2+}, \mathrm{Na}^{+}$, viscosity, sedimentation rate, etc. Overall, most of the 
variables of the quantity of blood cells and the concentration of electrolytes, hormones and metabolites display daily rhythmic profiles [6]. Of the blood variables, the level of plasma phosphate displays an overt diurnal rhythmicity with the nadir approximately at 11:00 AM, and the peak approximately at 12:30 AM [7].

In modern society, a substantial proportion of population (approximately $15-20 \%$ ) lives and works under schedules deleterious to their circadian rhythms [8]. Although numerous medical and ergonomical problems have been reported, rotating shift work schedules are still commonly employed in industrial society including maritime tasks and the detrimental effects have not been sufficiently concerned. For instance, non-24 $\mathrm{h}$ and frequently rotating schedules are commonly used in maritime missions [8-12]. Circadian rhythms display certain adaptation to environmental cues with a period in a range deviated from $24 \mathrm{~h}$. Within this range, the rhythms changes in accordance to the cues; beyond this range, the rhythms show a superposed profile of the cues and endogenous period [10].

Changes in sleep/wake status are accompanied by changes in gene expression, and vice versa. At the molecular level, circadian clock controls the expression and function of protein-coding genes and microRNAs (miRNAs) which are miRNAs are small, 20- to 22-nt non-coding RNA transcripts that base pair with mRNA to degrade it or inhibit its translation $[13,14]$. Insufficient sleep elicits expression changes in serum miRNAs targeting circadian rhythm and sleep homeostasis associated genes [15-17]. MiRNAs are also involved in the regulation of sleep diseases, e.g., narcolepsy and idiopathic hypersomnia [18]. On the other hand, miRNAs play a conserved role in regulating sleep and circadian clock in human and Drosophila [19-22]. These evidence demonstrate that miRNAs are involved in the regulation of circadian clock, and vice versa, misalignment in circadian rhythms may affect the expression and subsequent the function of a subset of miRNAs.

In this work, we collected the blood/plasma samples from subjects who participated in two experiments following a non-24-h schedule and a schedule with consecutive shift, respectively. The blood/plasma biochemical variables of these samples were measured and the serum miRNAs from one experiment were sequenced. And the findings revealed that extensive changes at the molecular and physiological levels due to circadian misalignment.

\section{Methods}

\section{Participants and bioethics}

Two experiments with volunteers were carried out in this study, one is called experiment $\# 1$ and the other \#2 for simplification. In experiment \#1, 15 healthy men (age, $23.1 \pm 0.8$ year; height, $175.7 \pm 4.8 \mathrm{~cm}$; weight, $73.5 \pm 7.2 \mathrm{~kg}$; data are means \pm SD) volunteered. No alcohol, smoking or caffeinated drinks and tea were allowed during this experiment. In experiment \#2, the volunteers included 12 healthy men (age, $32.0 \pm 4.4$ year; height, $173.1 \pm 4.6 \mathrm{~cm}$; weight, $70.5 \pm 9.8 \mathrm{~kg}$; data are means $\pm \mathrm{SD}$ ). No alcohol and 
smoking were allowed, and caffeinated drinks and tea were allowed only during the experiment period but not control and recovery periods in this experiment.

\section{Protocols and arrangements}

In experiment \#1, the participants were divided into three groups and worked on a 3-day shift rotation 3day shift rotation. The arrangement of experiment \#2 contained three periods: 1) control for 7 days; 2 ) 3-d rotatory shift for 30 days. The total sleep duration in every 3 days was $24 \mathrm{~h}$ though the time of sleep and waking up changed every day, which caused highly frequent shifts; 3 ) recovery for 7 days. In control and recovery periods, the participants lived on a fixed schedule with a period of 24-h.

The experiment \#2 constituted of four measurement time blocks: 1) the control from 8:00 AM on the first day to 10:00 PM on the second day; 2) the sleep deprivation (SD) period from 6:00 AM on the second day to $10: 00$ PM on the third day; 3 ) the $8 \mathrm{~h}$-on and $4 \mathrm{~h}$ - off schedule: from 6:00 AM on the fifth day to 2:00 AM on the eighth day. In control and recovery periods, the participants lived on a fixed schedule with a 24$\mathrm{h}$ period.

In experiment \#1, saliva was sampled every $4 \mathrm{~h}$ in control (day 2-3), early (days 20-21) and late (days 33-34) in experiment, respectively (Fig. 1A). The time of blood drawing after overnight fasting was between 7:00 AM and 8:00 AM for all of the subjects in control and recovery periods in both experiments. In experiment \#1, blood drawing was performed on day 7 in control and day 2 in recovery. In experiment \#2, blood drawing was performed on the days 2 and 3 in control and the days 1 and 2 in recovery.

\section{Measurement of salivary cortisol levels}

Salivette tubes (Sarstedt, Numbrecht, Germany; 51.1534.500) were used for saliva sampling for cortisol analysis. The cortisol level of $100 \mu \mathrm{l}$ salivary at each time point was measured with LC-MS/MS (TSQ Quantum Ultra), which included an ultimate 3000 system and a thermo scientific TSQ quantum access triple stage quadrupole mass spectrometer (Thermofisher, USA].

\section{Biochemical analysis of blood/serum samples}

The whole blood samples derived from experiment \#1 and the serum samples from experiment \#2 were subject to biochemical analysis, $100 \mu \mathrm{l}$ for each sample. AU5800 Series Chemistry Analyzers (Beckman Coulter, USA) was used for the analysis.

\section{Transcriptomic analysis of serum exosomal miRNAs}

One hundred $\mu \mathrm{l}$ of serum samples from each subject were pooled and the pooled sera were used for biochemical analysis. miRNA was isolated with TreSeq Small RNA Sample Prep Kits (Illumina, San Diego, USA), and ligated with adapters. After RT-PCR amplification and electrophresis, RNAs between $22 \mathrm{nt}$ and $30 \mathrm{nt}$ were excised and purified for library construction. The sequencing was performed on Illumina Hisseq2000/2500. The read of single end was $50 \mathrm{bp}$. 
Raw reads were subjected to an in-house program, ACGT101-miR (LC Sciences, Houston, Texas, USA) to remove the sequences of adapter dimers, junk, low complexity, common RNA families (rRNA, tRNA, snRNA, snoRNA) and repeats. The significance threshold was set to be 0.01 and 0.05 . To predict the genes targeted by most abundant miRNAs, two computational target prediction algorithms (TargetScan 50 and Miranda 3.3a) were used to identify miRNA binding sites $[23,24]$. Finally, the data predicted by both algorithms were combined and the overlaps were calculated. The KEGG Pathways of these most abundant miRNAs, miRNA targets were also annotated.

\section{Statistics}

In the analysis of blood/serum biochemical parameters, the results are presented as the means $\pm S D$. Wilcoxon matched-pairs signed rank test was used to analyze the values as indicated at certain time points. In the analysis of miRNA sequencing data, differential expression of miRNAs based on normalized deep-sequencing counts was analyzed by selectively using Fisher exact test, Chi-squared 2X2 test, Chi-squared $\mathrm{nXn}$ test, Student $t$ test, or ANOVA according to the protocol. The significance of qRTPCR results was detected by Student's t test. * represents the $P$-value of the statistical tests being less than a significance level of $0.05(P<0.05) ; * \star P<0.01$, and $\# P<0.001$. Some of the samples failed to be detected which are not included in the analysis.

\section{Results}

\section{Rotatory schedule led to changes in the levels and rhythms of blood cortisol}

Shift work and jet lag have been shown to affect circadian rhythms in many studies [25]. Shift work is common in steel industries, petroleum industries, power plants, medicine and nursing, police forces and maritime $[12,26]$. Shift work is also one type of social jet lag. According to the 3rd international classification of sleep disorders (ICSD-3), the comprehensive inadaptations and disorders caused by shift schedules are defined as shift-work disorders [27].

A schedule with frequent shift was used in experiment \#1 (Fig. 1A), as detail described in the section of methods. We sampled and measured the levels salivary cortisol in two consecutive days in control, early and late stages, respectively. The saliva sampling was conducted every $4 \mathrm{~h}$ in these time blocks. The results revealed that the serum cortisol levels displayed daily rhythms in both control and experiment periods (Fig. 1B,C). The peaking time of cortisol which represents the circadian phase was overtly delayed in the early and late stages compared to control (Fig. 1C). The cortisol levels in the early stage were significantly elevated (Fig. 1D). And consistently, the amplitude in the early stage but not the late stage during experiment was significantly elevated (Fig. 1E).

\section{Changes in whole blood biochemical variables due to frequent shift}


We next measured the changes in the whole blood biochemical variables of the subjects following the rotatory schedule. The results revealed significant changes of many serum variables in recovery (after rotatory schedule) compared to that in control (before rotatory schedule). The increased serum variables included: glucose concentration, phosphate ion concentration, cholesterol levels, creatinine levels, direct bilirubin levels, low density lipoprotein levels, gamma-glutamyl transferase levels, total protein levels, albumin levels, superoxide dismutase levels, alanine transaminase levels, alkaline phosphatase levels, glutamic oxalacetic transaminase levels, Apolipoprotein-B levels,. By contrast, the variables of homocysteine levels, urea levels, triglyceride levels, total bilirubin levels, showed significant decrease after the experiment (Fig. 3; Supplemental table 2). The average levels of serum phosphate were 1.193 \pm 0.152 in control and $1.343 \pm 0.099$, respectively (Fig. 3c; supplemental table 2). The serum variables showed no significant change included iron ion concentration, iron ion concentration, potassium ion concentration, etc, which are listed in supplemental Table 1.

\section{Changes in plasma biochemical variables due to non-24-h schedule}

To further address the effects of circadian misalignment on blood/serum variables, we recruited volunteers and conducted experiment \#2, in which the volunteers followed an $8 \mathrm{~h}$ on and $4 \mathrm{~h}$ off schedule (Fig. 3A). The non-24 $\mathrm{h}$ schedules are commonly used in maritime tasks, including maritime oil \& gas mining, commercial vessel, submarine and other fleets $[9,11]$. A number of studies have demonstrated that $18 \mathrm{~h}$ or $12 \mathrm{~h}$ work schedules are deleterious to health and performance [8-11]. Although experiment \#1 contained a period of 36-h SD, sleep time was compensated with after SD which can be supported by results from the psychomotor vigilance test, therefore, the effects of SD could be neglected [11].

In experiment \#2, a number of serum variables showed significant changes in recovery (after non-24 $\mathrm{h}$ schedule) compared to control (before non-24 h schedule). The increased serum variables include: phosphate levels, total bile acids levels, triglyceride levels, prealbumin levels, aspartic aminotransferase levels, apolipoprotein A-1 levels, absolute neutrophil count, red blood cell distribution width, and white blood cell counts. By contrast, the variables of sodium ion concentration, iron ion concentration, total protein levels, albumin levels, total bilirubin levels, showed significant decrease after the experiment (Fig. 2; Supplemental table 1). Approximately $90 \%$ of phosphate $(\mathrm{Pi})$ is present within the skeleton, $10 \%$ in the soft tissue and less than $1 \%$ in the serum [Hernando et al 2019]. In experiment \#1, the average levels of serum phosphate were $1.210 \pm 0.141$ in control and $1.330 \pm 0.117$, respectively (Fig. 3b; supplemental table 2). The serum variables showed no significant change, including potassium ion concentration, chlorine ion concentration, glucose concentration, etc (supplemental Table 2). It is noteworthy that significant elevation in phosphate was observed in both experiments, suggesting it is of high risk due to disturbance in the circadian rhythms (Fig. 2,3; supplemental tables 1,2).

\section{Transcriptomic analysis of serum exosome miRNAs}

We conducted miRNA sequencing using the pooled serum samples derived from experiment \#1, which identified 1008 and 1172 miRNAs in control and recovery, respectively. The control and recovery samples 
contain 852 miRNAs in common (Fig. 4a). In total, 249 miRNAs $(P<0.01)$ and 395 miRNAs $(P<0.05)$ were identified to show differential expression before and after experiment, respectively (Fig. 4b,c; supplemental Table 3), and the differentially expressed miRNAs are implicated in comprehensive pathways, including multiple metabolic pathways, immunity, neural function (axon guidance) and cancer associated pathways (Fig. 4d).

We next looked at the changes in miRNAs targeting the core circadian clock genes, as rotatory schedule causes misalignment in circadian rhythms. Some miRNAs targeting PER1, PER2, CRY1, CRY2, BMAL 1/ARNTL, BMAL2/ARNTL2, RORA, RORB, NPAS2 and DEC1 genes were identified (Fig. 4e; supplemental Table 3). Quantitative reverse transcription polymerasec reaction (qRT-PCR) results confirmed the expression profiles revealed from sequencing (Fig. 4f-i). Together, these data suggest that comprehensive changes occurred in circadian clock associated miRNAs due to the shift schedule.

Insufficient sleep leads to changes in the amplitude of several set of genes associated with a variety of physiological processes [17]. In this study, expression changes in those miRNAs associated with sleep homeostasis were also observed, including miRNAs targeting STAT3, KCNV2, IL6 and CAMK2D (supplemental Table 3) [17].

Transport of Pi across the renal proximal tubule is a crucial step in maintaining Pi homeostasis. In kidney, sodium-dependent $\mathrm{Pi}(\mathrm{Na} / \mathrm{Pi})$ transport systems are localized in the brush-border membrane, and bulk of filtered $\mathrm{Pi}$ is reabsorbed in the proximal tubule. Sodium-dependent $\mathrm{Pi}(\mathrm{Na} / \mathrm{Pi})$ transport systems mediate the rate-limiting step in the Pi re-absorptive process. Three classes of Na/Pi cotransporters, NPTI, NPTII and NPTIII, have been identified in human. Of the NPT families, phosphate homeostasis is maintained by NPT2A members (including SLC34A1/NPT2A, SLC34A2/NPT2B and SLC34A3/NPT2C) in kidney and intestine at the organism level and SLC20A1/PiT1 and SLC20A2/PiT2 at the cellular level [28-30]. PHEX and FGF-23 are involved in regulation of Pi homeostasis and they have been identified in inherited and acquired hypophosphatemia [29]. We searched potential miRNAs targeting genes involved in the regulation of phosphate regulation, including miRNAs targeting NPTII genes (SLC34A1, SLC34A2 and SLC34A3), phosphate regulating gene with homologies to endopeptidases on the $\mathrm{X}$ chromosome (PHEX) and fibroblast growth factor-23 (FGF23) [29], however, no miRNAs targeting these genes were found.

\section{Discussion}

The glucocorticoid cortisol responds to stress and inflammation, and it is secreted by the hypothalamicpituitary-adrenal axis [Brown 2020]. In this work, we showed that a rotatory schedule led to delayed circadian phase in serum cortisol during experiment and increased circadian amplitude in serum cortisol during the early stage but not the late stage (Fig. 1B-D), suggesting that circadian rhythm is subject to change and stress occurs owing to the rotatory schedule.

The albumin level and total bilirubin level decreased in experiment \#1 but increased in experiment \#2, while the triglyceride level increased in experiment \#1 but decreased in experiment \#2 (Fig. 2,3; supplemental Tables 1,2), which may be owing to individual difference or reflect the different effects of 
between non-24 schedule and rotatory schedule. Changes in metal elements including sodium, iron were observed in experiment \#1. The levels of glucose, cholesterol, urea and creatinine showed changes in experiment \#2 (Fig. 3; supplemental Tables 2). These results suggest that these two schedules resulted in the extensive alterations in physiology and metabolism, although most of the changes are still within the normal ranges. However, in experiment \#1, the levels of bile acids of several subjects are higher than the normal range $(0.1 \otimes 10.0 \mu \mathrm{mol} / \mathrm{L})$ after the experiment (Fig. 2f; supplemental Table 1$)$, suggesting that risks of dysfunctions in liver or hepatic duct should be taken into consideration [31].

Inorganic phosphate is a vital component of bone mineralization, phospholipids in membranes, nucleotides that provide energy and serve as components of DNA and RNA, and phosphorylated intermediates in cellular signaling [32]. It is also required for skeletal development, mineral metabolism, and diverse cellular. The level of Serum $\mathrm{Pi}$ is maintained within a narrow range - the normal plasma phosphate concentration is $0.81-1.45 \mathrm{mmol} / \mathrm{L}$, which is regulated by multiple factors, e.g., intestinal absorption, exchange with intracellular and bone storage pools, renal tubular reabsorption, and their interactions [33, 34].

Plasma phosphate imbalance, including hypophosphatemia and hyperphosphatemia, are associated with effects on any organs. Occurrence of hyperphosphatemia is reported in renal failure, hemolysis, tumor lysis syndrome, and rhabdomyolysis [34]. Both human and animal studies reveal that calciumphosphate homeostatic abnormalities may be associated with cardiovascular disease, and hyperphosphatemia and hypercalcemia to be cardiovascular events [35]. However, we did not find miRNAs targeting genes regulating phosphate homeostasis, suggesting that the changes in serum phosphate level may be independent of serum miRNAs.

Kidney regulates the maintenance of fluid and ion homeostasis through filtering the blood and blood pressure as consequence [36, 37]. Circadian clocks genes have been demonstrated to govern kidney physiology and function at the molecular level [37]. Therefore, circadian misalignment might affect the kidney function which accounts for the imbalance in serum phosphate.

miRNAs constitute a post-transcriptional layer in the regulation of circadian clock [38, 39]. Insufficient sleep causes changes in the expression amplitude of human blood transcritome [17], suggesting that impairment in circadian rhythms and sleep may cause transcriptomic alterations. In addition to phosphate metabolism, in this work we identified a substantial set of functional miRNAs which are implicated in multiple pathways or physiological processes, including those targeting genes regulating circadian clock and sleep homeostasis, suggesting that these miRNAs may contribute to the misalignment in circadian rhythms and sleep homeostasis caused by abnormal work schedules. The changed miRNAs may be causative or resultant of the desynchronized circadian rhythms. More possibly, these two factors may be tightly associated and mutually influence each other. In addition, miRNAs associated with cancer, neural function and several metabolic pathways were also identified, which suggests that desynchronized circadian rhythms may increase the risks of many disorders through modulating the expression of corresponding miRNAs. 
In both experiments, the participants lived in isolation condition, and most of the environmental cues including lighting, noise, ambient temperature and workload were controllable so that we could focus on the effects of circadian misalignment. Instead, in real condition like submarine, as the microenvironment differs between different locations or posts on board, the effects of circadian misalignment might be hard to be addressed due to inconsistency between different micro environmental domains [12].

\section{Conclusions}

In this work, our findings would add important evidence that sleep deprivation, non- $24 \mathrm{~h}$ schedules and shift schedules may lead to remarkable changes in circadian rhythms of cortisol and blood variables. Interestingly, we found that the level blood phosphate was increased in both experiments, suggesting that disruption of circadian rhythms may cause comprehensive effects through altering the phosphate metabolism. Furthermore, circadian misalignment may elicit physiological stress.

Circulating microRNA profile has been proposed as a potential biomarker for obstructive sleep apnea diagnosis [40]. We found that a subset of plasma miRNAs are involved in circadian rhythms, sleep homeostasis and phosphate metabolism, which may better the investigation of the underlying molecular mechanisms of circadian misalignment and serve as biomarkers in further study. Together, these findings would help understand the deleterious effects of shift schedules and improve the welfare and performance of the personnel who live and work with rotatory non-24-h schedules.

\section{Abbreviations}

RT-PCR: reverse transcriptase-polymerase chain reaction; miRNA: microRNA; SD: sleep deprivation; PHEX: phosphate regulating gene with homologies to endopeptidases on the $X$ chromosome; FGF-23: fibroblast growth factor-23; Pi: phosphate.

\section{Declarations}

\section{Authors' contributions}

$Y S, Y Y, X W, L Z, Z T$ and JG designed and organized the experiments. GH, HM, SL, TZ, XZ and HJ performed experiments. SL, XM, XG, SC and HY helped with data collection and statistical analysis. All authors read and approved the final manuscript.

\section{Funding}

This work was supported by the NDSTISZP (1716312ZT00210001), the Open Fund of the National Key Laboratory of Human Factors Engineering in the Astronaut Center of China (SYFD180051809K), the National Natural Science Foundation of China (Grants 31871188) and the Space Medical Experiment Program (HYZHXM03007). 
Acknowledgements

We thank all of the participants for their contribution to these experiments.

\section{Availability of data and materials}

Not applicable.

\section{Ethics approval and consent to participate}

The research methods and procedures of experiment \#1 were in accordance with the guidelines and approved by the Ethics Committee of China Institute of Marine Technology \& Economy. All participants provided written informed consent. The research methods and procedures of experiment \#2 were in accordance with the guidelines and approved by the Ethics Committee of the Sixth Medical Center, General Hospital of Chinese People's Liberation Army. All participants provided written informed consent.

\section{Consent for publication}

Not applicable.

\section{Competing interests}

The authors declare that they have no competing interests.

\section{References}

1. Dunlap J, Loros J. Yes, circadian rhythms actually do affect almost everything. Cell Res. 2016;26(7):759-60.

2. Bechtold DA, Gibbs JE, Loudon AS. Circadian dysfunction in disease. Trends Pharmacol Sci. 2010;31(5): 191-8.

3. Wulff K, Gatti S, Wettstein JG, Foster RG. Sleep and circadian rhythm disruption in psychiatric and neurodegenerative disease. Nat Rev Neurosci. 2010;11(8):589-99.

4. Luce GG. Biological rhythms in human and animal physiology. Dover Publications. New York;1971. P.59.

5. Aschoff J. Handbook of behavioral neurobiology. Biological rhythms. Volume 4. Plenum Press. New York and London; 1981. p.3-5.

6. O'Neill JS, Reddy AB. Circadian Clocks in Human Red Blood Cells. Nature. 2011;469(7331): 498-503.

7. Subramanian R, Khardori R. Severe hypophosphatemia. Pathophysiologic implications, clinical presentations, and treatment. Medicine. 2000;79(1):1-8.

8. Chellappa SL. Circadian misalignment: A biological basis for mood vulnerability in shift work. Eur J Neurosci. 2020; 52(8):3846-50. 
9. Colquhoun WP, Rutenfranz J, Goethe H, Neidhart B, Condon R, Plett R, Knauth P. Work at sea: a study of sleep, and of circadian rhythms in physiological and psychological functions, in watchkeepers onmerchant vessels. I. Watchkeeping on board ships: a methodological approach. Int Arch Occup Environ Health. 1988;60: 321-29.

10. Guo JH, Qu WM, Chen SG, Chen XP, Lv K, Huang ZL, et al. Keeping the right time in space: importance of circadian clock and sleep for physiology and performance of astronauts. Mil Med Res. 2014;1:23.

11. Ma H, Li Y, Liang H, Chen S, Pan S, Chang L, et al. Sleep deprivation and a non-24 h working schedule lead to extensive alterations in physiology and behavior. FASEB J. 2019;33(6):6969-79.

12. Trousselard $M$, Leger $D$, van Beers $P$, Coste $O$, Vicard $A$, Pontis $J$, et al. Sleeping under the ocean: despite total isolation, nuclear submariners maintain their sleep and wake patterns throughout their under sea mission. PLoS One. 2015;10(5): e0126721.

13. Hicks SD, Khurana N, Williams J, Greene CD, Uhlig R, Middleton FA. Diurnal oscillations in human salivary microRNA and microbial transcription: Implications for human health and disease. PLoS One. 2018;13(7):e0198288.

14. Koike N, Yoo SH, Huang HC, Kumar V, Lee C, Kim TK, et al. Transcriptional architecture and chromatin landscape of the core circadian clock in mammals. Science. 2012;338(6105):349-54.

15. Davis CJ, Bohnet SG, Meyerson JM, Krueger JM. Sleep loss changes microRNA levels in the brain: a possible mechanism for state-dependent translational regulation. Neurosci Lett. 2007;422(1):68-73.

16. Davis CJ, Clinton JM, Krueger JM. MicroRNA 138, let-7b, and 125a inhibitors differentially alter sleep and EEG delta-wave activity in rats. J Appl Physiol. 2012;113(11):1756-62.

17. Möller-Levet CS, Archer SN, Bucca G, Laing EE, Slak A, Kabiljo R, et al. Effects of insufficient sleep on circadian rhythmicity and expression amplitude of the human blood transcriptome. Proc Natl Acad Sci U S A. 2013;110(12):E1132-41.

18. Holm A, Bang-Berthelsen CH, Knudsen S, Kornum BR, Modvig S, Jennum P, et al. miRNA Profiles in Plasma from Patients with Sleep Disorders Reveal Dysregulation of miRNAs in Narcolepsy and Other Central Hypersomnias. Sleep. 2014;37(9):1525-33.

19. Liang X, Zhang L, Wan Y, Yu X, Guo Y, Chen X, et al. Changes in the Diurnal Rhythms during a 45-Day Head-Down Bed Rest. PLoS One. 2012;7(10):e47984.

20. Wan Y, Zhang L, Yu X, Guo Y, Yu J, Li H, et al. Influence of 45 d $-6^{\circ}$ Head-down Bed Rest on Concentration and Circadian Rhythms of Urinal Calcium and Phosphate. Space Med \& Med Eng. 2015;28(1):11-5.

21. Vodala S, Pescatore S, Rodriguez J, Buescher M, Chen YW, Weng R, et al. The oscillating miRNA 959964 cluster impacts Drosophila feeding time and other circadian outputs. Cell Metab. 2012;16(5):601-12.

22. Goodwin PR, Meng A, Moore J, Hobin M, Fulga TA, Vactor DV, et al. MicroRNAs Regulate Sleep and Sleep Homeostasis in Drosophila. Cell Rep. 2018;23(13): 3776-86. 
23. Agarwal V, Bell GW, Nam JW, Bartel DP. Predicting effective microRNA target sites in mammalian mRNAs. Elife. 2015;4:e05005.

24. Betel D, Wilson M, Gabow A, Marks DS, Sander C. The microRNA.org resource: targets and expression. Nucleic Acids Res. 2008;36(Database issue):D149-53.

25. Reid KJ, Abbott SM. Jet Lag and Shift Work Disorder. Sleep Med Clin. 2015;10(4):523-35.

26. Akbari H, Mirzaei R, Nasrabadi T, Gholami-Fesharak M. Evaluation of the Effect of Shift Work on Serum Cholesterol and Triglyceride Levels. Iran Red Crescent Med J. 2015;17(1): e18723.

27. Sack RL, Auckley D, Auger RR, Carskadon MA, Wright KP Jr, Vitiello MV, et al; American Academy of Sleep Medicine. Circadian rhythm sleep disorders: part I, basic principles, shift work and jet lag disorders. An American Academy of Sleep Medicine review. Sleep. 2007;30(11):1460-83.

28. Hernando N, Gagnon KB, Lederer ED. Phosphate Transport in Epithelial and Nonepithelial Tissue. Physiol Rev. 2020;doi: 10.1152/physrev.00008.2019.

29. Takeda E, Taketani Y, Sawada N, Sato T, Yamamoto $H$. The regulation and function of phosphate in the human bod Biofactors. 2004;21(1-4):345-55.

30. Tenenhouse HS. Recent advances in epithelial sodium-coupled phosphate transport. Curr Opin Nephrol Hypertens. 1999;8(4):407-14.

31. Sharma KR. Review on bile acid analysis. Int J Pharm Biomed Sci. 2012;3(2):28-34.

32. Lederer E. Regulation of serum phosphate. J Physiol. 2014;592(Pt 18): 3985-95.

33. Shiber JR, Mattu A. Serum phosphate abnormalities in the emergency department. J Emerg Med. 2002;23(4):395-400.

34. Berner YN, Shike M. Consequences of phosphate imbalance. Annu Rev Nutr. 1988;8:121-48.

35. Slinin Y, Foley RN, Collins AJ. Calcium, phosphate, parathyroid hormone, and cardiovascular disease in hemodialysis patients: The USRDS waves 1, 3, and 4 study. J Am Soc Nephrol. 2005;16:1788-93.

36. Ede MC, Faulkner MH, and Tredre BE. An intrinsic rhythm of urinary calcium excretion and the specific effect of bedrest on the excretory pattern. Clin Sci. 1972;42(4):433-45.

37. Solocinski K, Gumz ML. The Circadian Clock in the Regulation of Renal Rhythms. J Bio Rhythms. 2015;30(6):470-86.

38. Nandi A, Vaz C, Bhattacharya A, Ramaswamy R. miRNA-regulated dynamics in circadian oscillator models. BMC Syst Biol. 2009;3:45.

39. O'Neill JS, Hastings MH. Circadian clocks: timely interference by microRNAs. Curr Biol. 2007;17(17):R760-2.

40. Santamaria-Martos F, Benítez I, Ortega F, Zapater A, Giron C, Pinilla L, et al. Circulating microRNA profile as a potential biomarker for obstructive sleep apnea diagnosis. Sci Rep. 2019;9(1):13456.

\section{Figures}


A

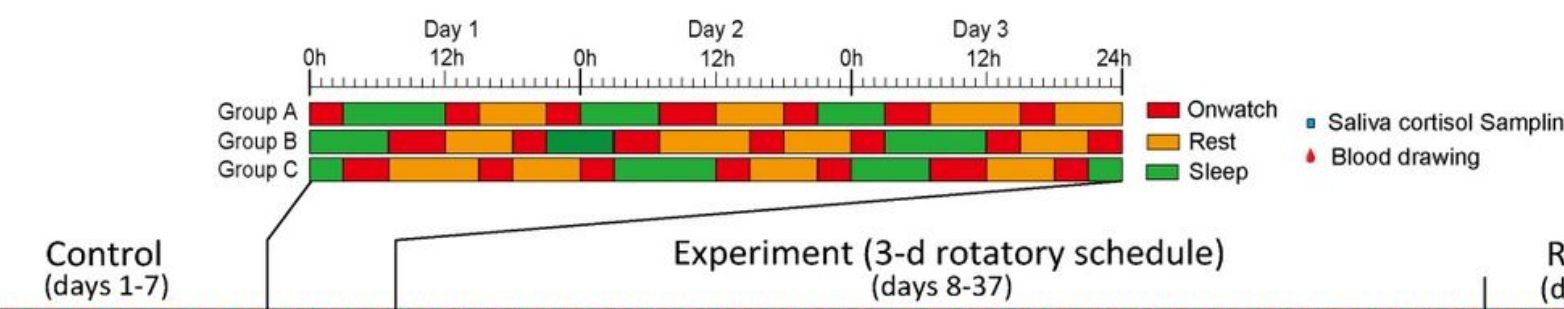

Recovery

(days 38-44)

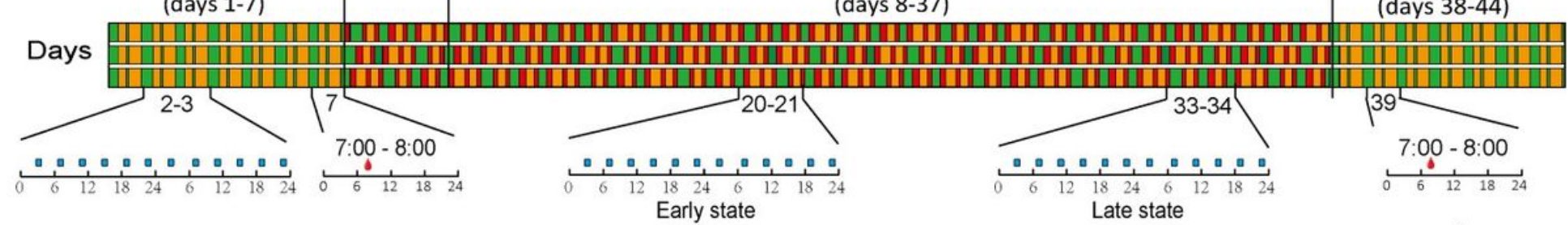

B

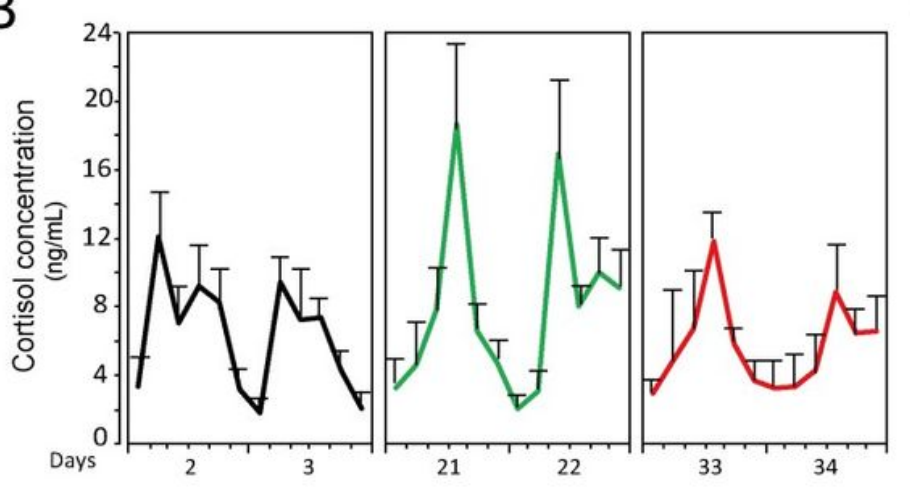

C

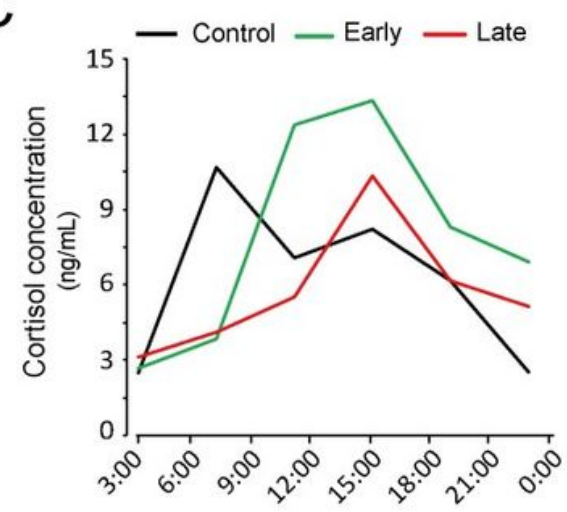

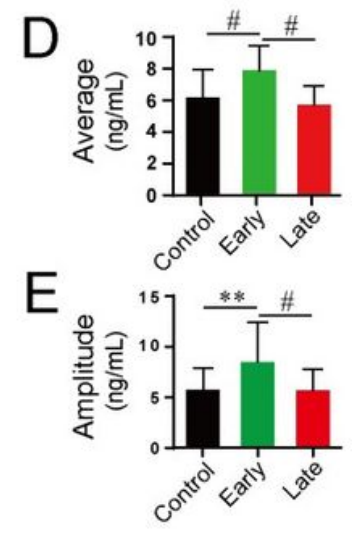

Figure 1

Experimental setup, arrangement. A Diagram of the experiment \#1. The time points of blood drawing and saliva sampling are denoted. A 3-d rotatory schedule is shown (days 1-3) in a zoomed view as example. Legends are labeled on the top. B Oscillation of serum cortisol levels in periods of control and 3-d rotatory schedule. C Comparison of the peaking time of cortisol levels. The levels of salivary cortisol were averaged and displayed in a 24-h course. D Average of cortisol levels in control, early and late periods in experiment. Data are mean $\pm S E . n=12$. E Relative amplitude values of cortisol. The results are presented as the mean values of every $4 \mathrm{~h}$. Data are mean $\pm S E . ~ n=12$. 

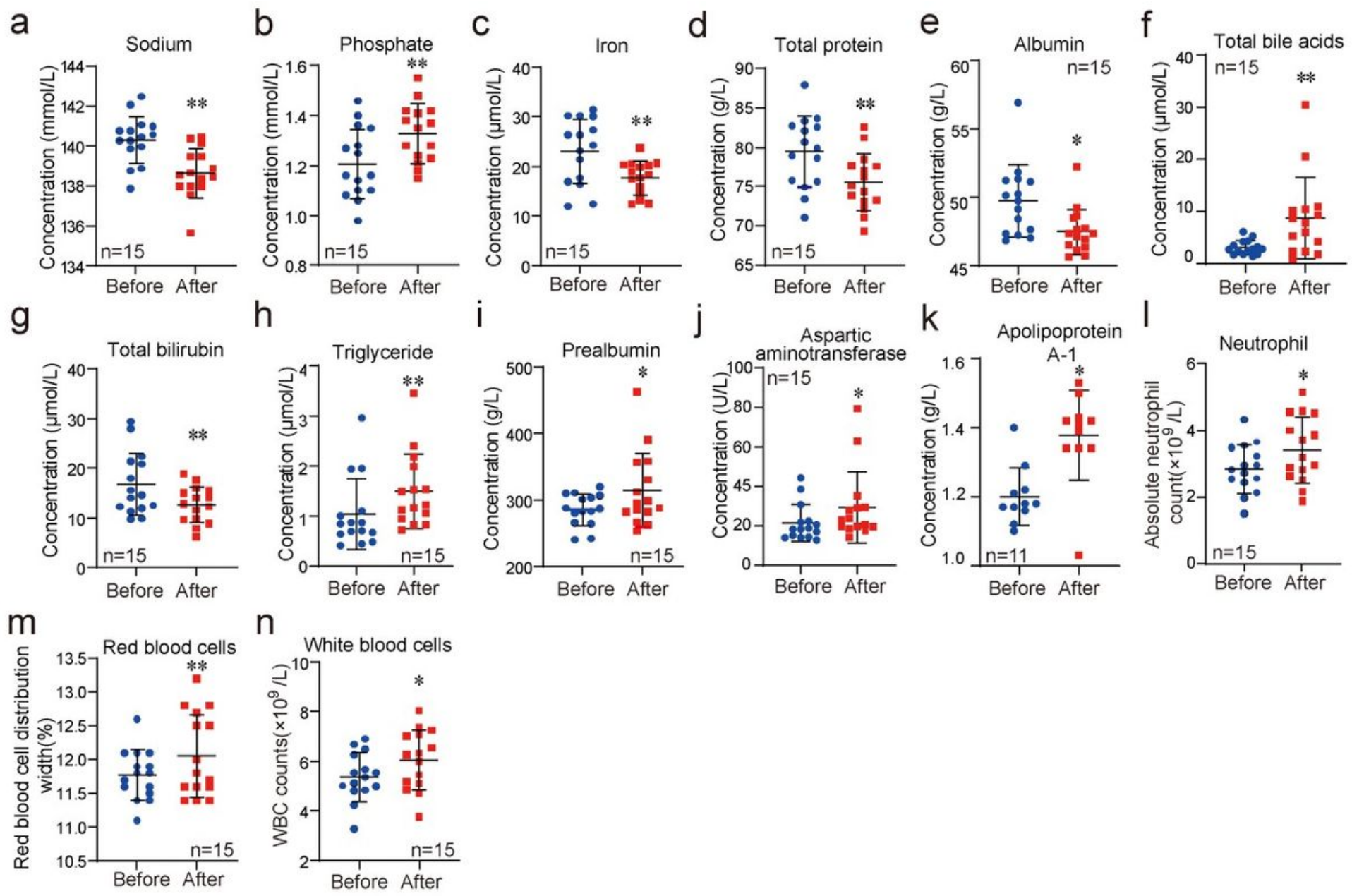

\section{Figure 2}

Results of blood biochemical analysis. Scatter plots with bars of blood parameters from experiment \#1 in control (Ctrl) and recovery. Panels a to s are the results of $\mathrm{Na}+(\mathrm{A})$, Phosphate (B), Fe (C), serum total protein $(D)$, serum albumin $(E)$, total serum bile acids $(F)$, serum total bilirubin $(G)$, serum triglyceride $(H)$, serum prealbumin $(I)$, serum aspartic aminotransferase $(J)$, serum apolipoprotein $A-1(K)$, neutrophil $(L)$, red blood cells $(M)$, white blood cells $(N)$. Data are mean $\pm S E$. A-J, L-N: $n=15 ; K: n=11 .{ }^{*} P<0.05, * * P<$ 0.01 . 
A

$\square$ unrecorded $\square$ Sleep $\square$ Rest / work

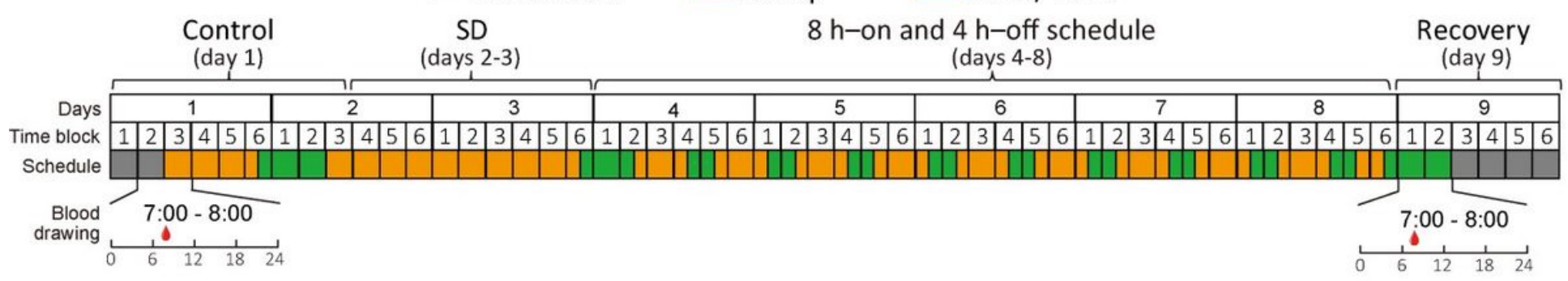

B

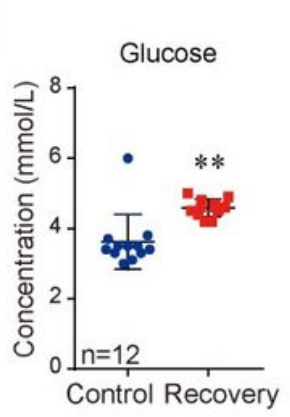

$\mathrm{H}$

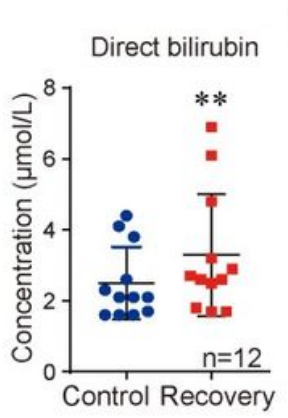

$\mathrm{N}$

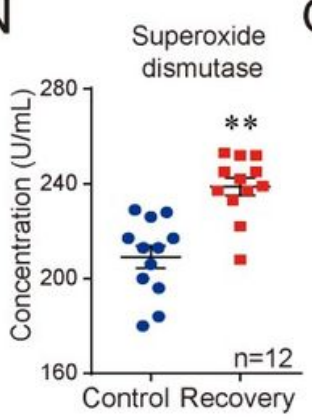

C
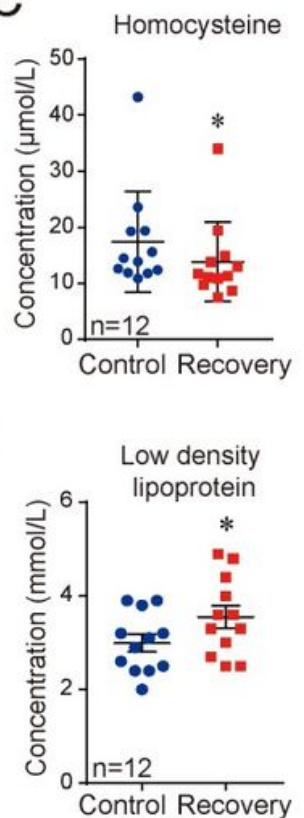

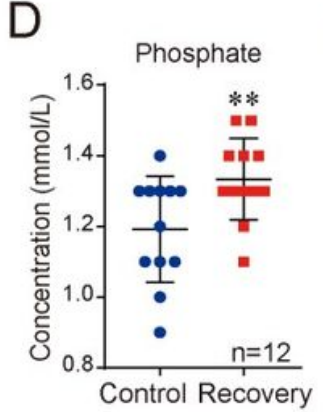

E
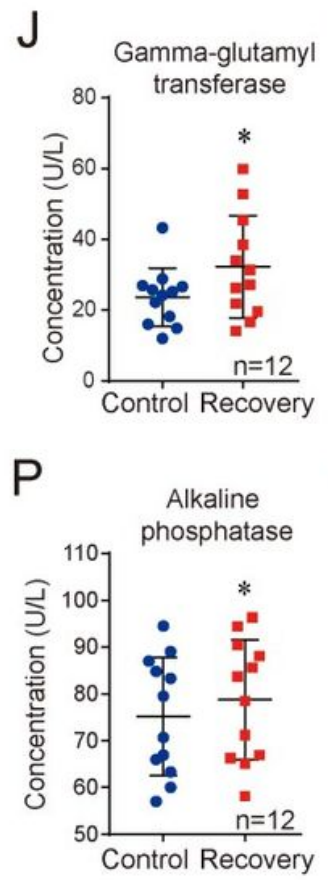

F
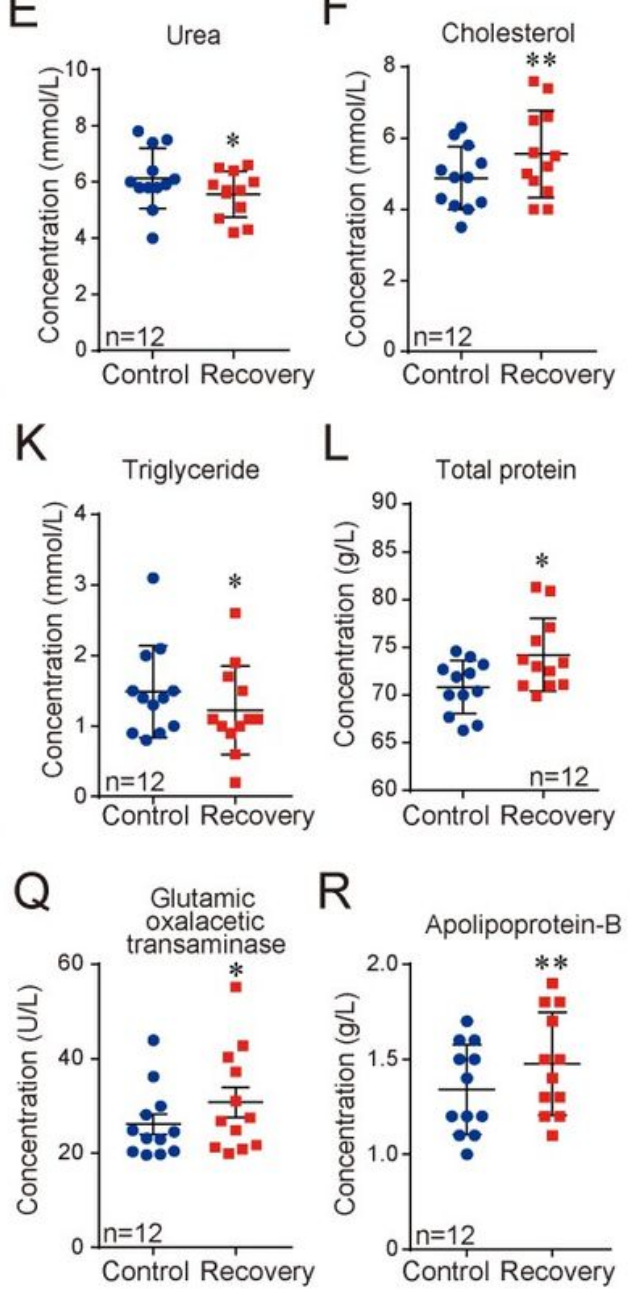

G

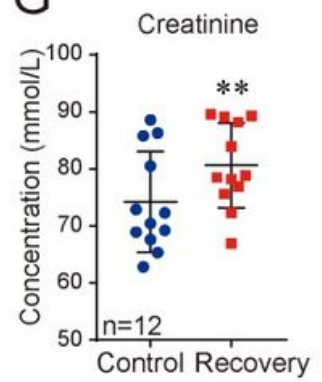

M

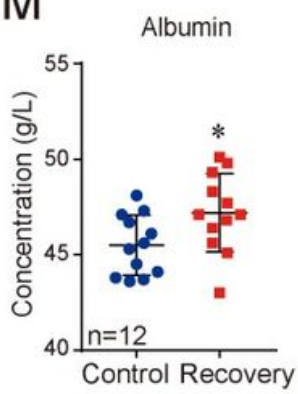

$S$

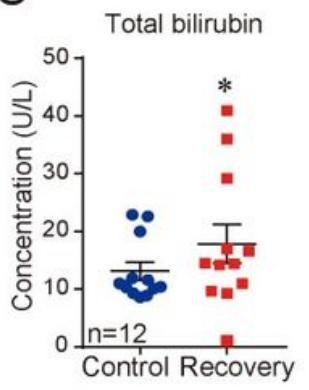

Figure 3

Results of plasma biochemical analysis. Scatter plots with bars of blood parameters from experiment \#2 in control (Ctrl) and recovery. A Diagram of the experiment \#2. The time points of blood drawing in control are indicated. Every day is divided into six equal time blocks (1-6) and the duration of each is $6 \mathrm{~h}$. Legends are labeled on the top. Panels a to $s$ are the results of glucose (B), homocysteine (C), phosphate $(D)$, urea $(E)$, cholesterol $(F)$, creatinine $(G)$, DBIL $(H)$, low density lipoprotein $(I)$, GGT $(J)$, triglyceride $(K)$, total protein $(L)$, albumin $(M), S D O(N)$, ALT $(O)$, ALP $(P), A S T(Q)$, APOB $(R)$, total bilirubin (S). Data are mean \pm SE. $n=12$. ${ }^{*} P<0.05, * * P<0.01$. 


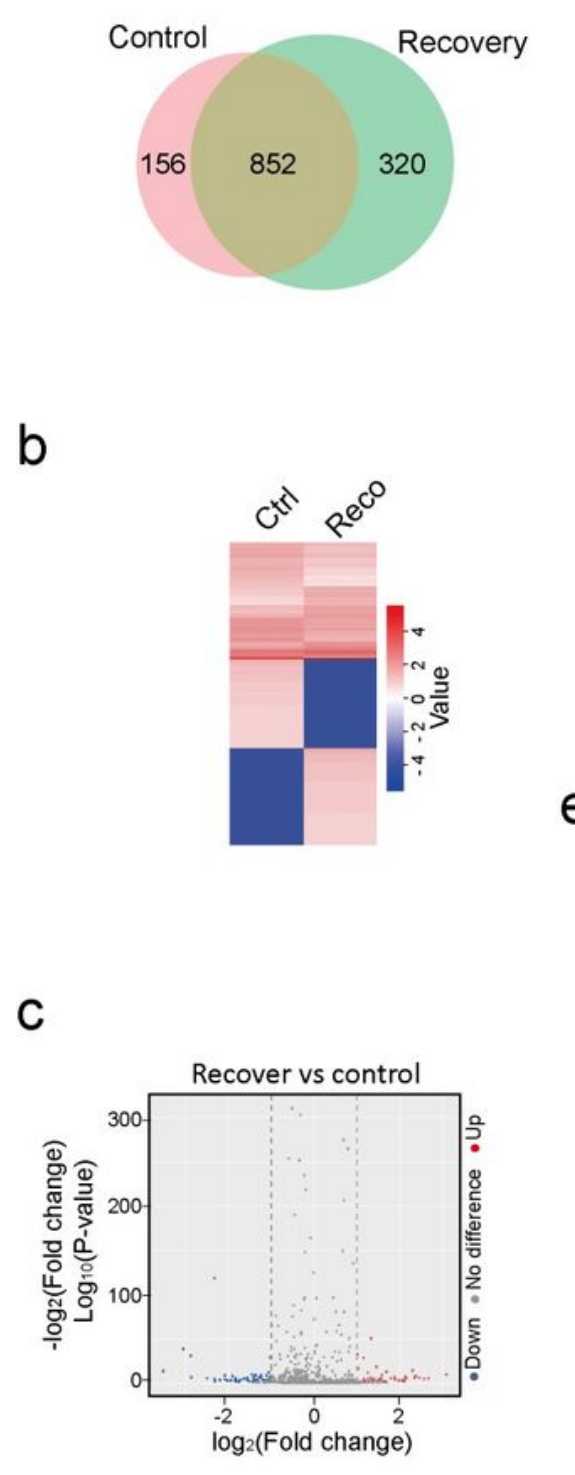

d

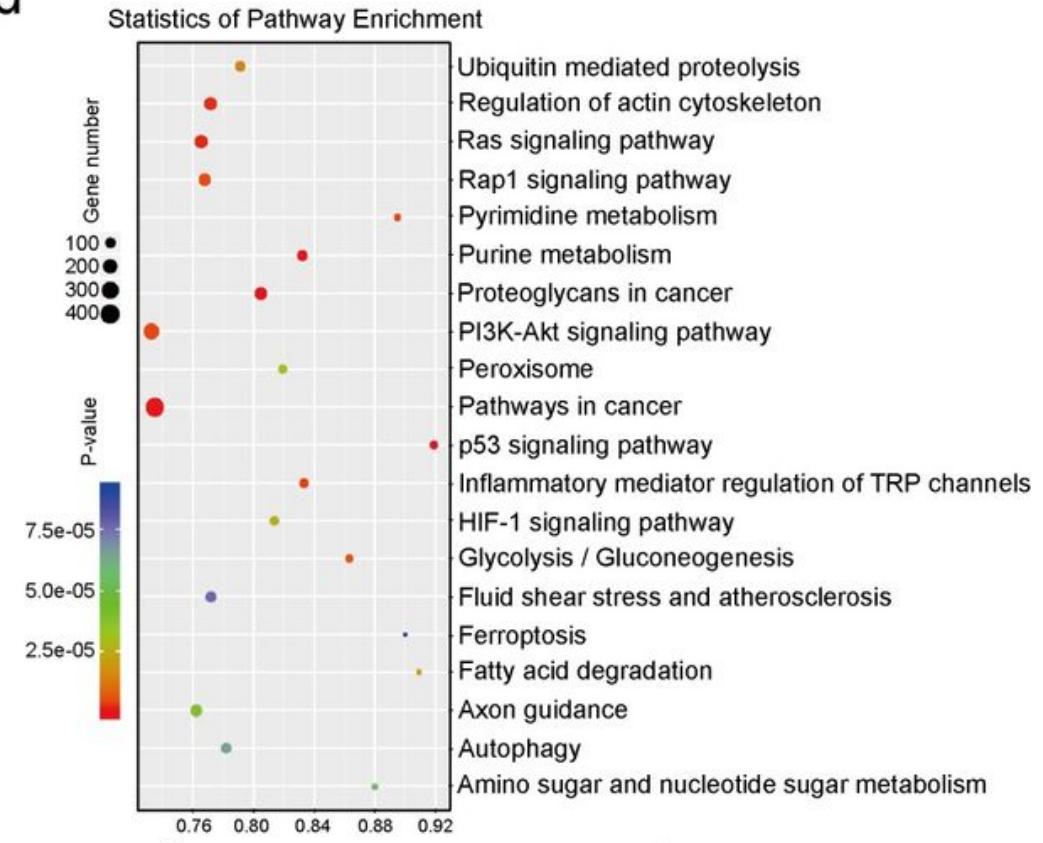

e

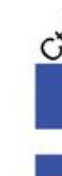

\section{hsa-miR-1197}

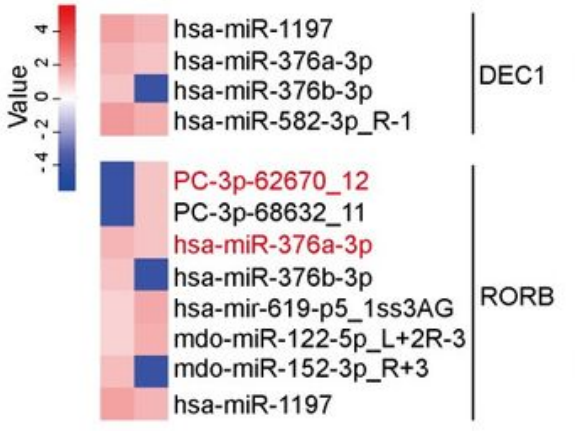

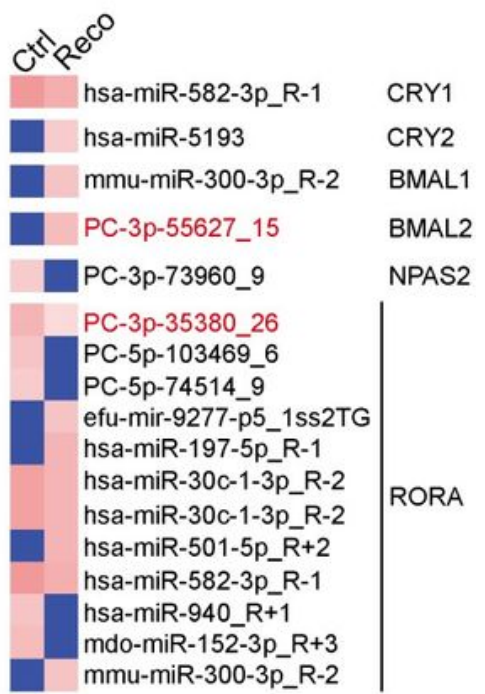
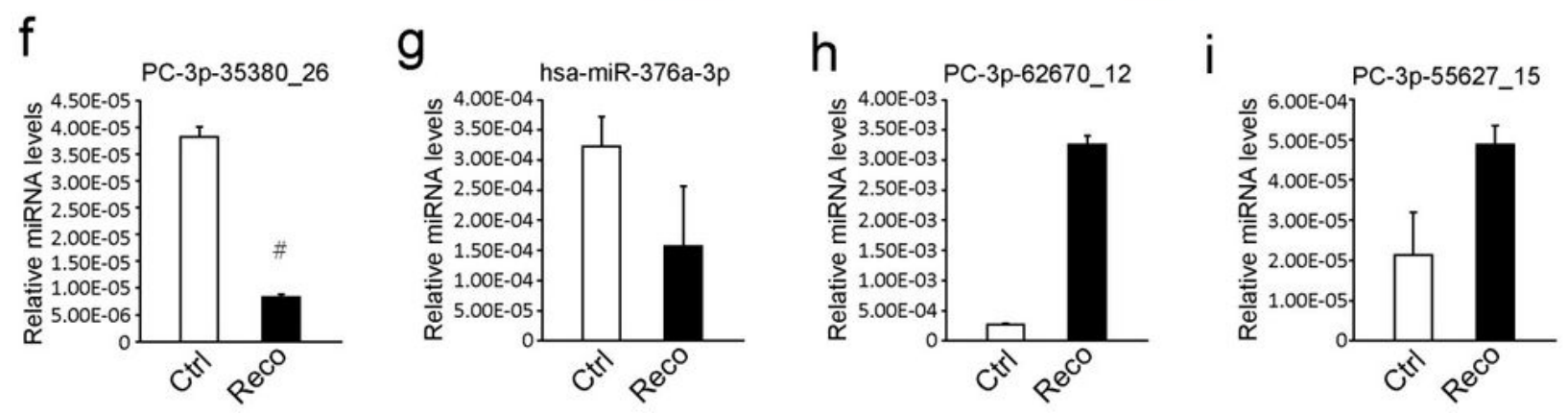

\section{Figure 4}

Transcriptomic analysis of plasma exosomal miRNAs. A Venn diagram of expressed miRNAs in pooled sera in each group. B Heat map of all miRNAs showing changed levels in control (Ctrl) and recovery (Reco). $P<0.01$. C,D KEGG enrichment scatter plot of differentially expressed miRNAs. E Heat map of examples of differentially expressed serum miRNAs targeting core circadian clock genes between control (Ctrl) and recovery (Reco). Only those differential miRNAs with P values $<0.05$ are shown. Four miRNAs 
in red were subjected to qRT-PCR validation. F-L q-RT-PCR validation of four indicated miRNAs. Data are mean \pm SE. $n=7$. * $P<0.05$, ** $P<0.01$, \# $P<0.001$.

\section{Supplementary Files}

This is a list of supplementary files associated with this preprint. Click to download.

- Supplementaltable1.xlsx

- Supplementaltable2.xlsx

- Supplementaltable3.xlsx 